\title{
Agentes causantes de bacteriemia en niños con cáncer y neutropenia febril de alto riesgo en seis hospitales de Santiago, Chile, período 2004- 2009
}

\author{
Yanahara Solís, Ana M. Álvarez, David Fuentes, Daniela de la Barra, Carmen L. Avilés, \\ Ana Becker, Carmen Salgado, Pamela Silva, Santiago Topelberg, Juan Tordecilla, Mónica Varas, \\ Milena Villarroel, Tamara Viviani, Marcela Zubieta, Sócrates Aedo y María E. Santolaya
}

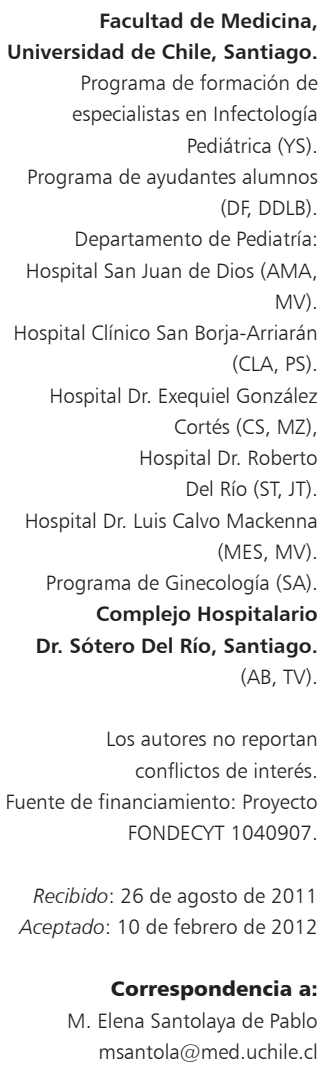

Facultad de Medicina, Universidad de Chile, Santiago.

Programa de formación de especialistas en Infectología Pediátrica (YS). Programa de ayudantes alumnos (DF, DDLB)

Departamento de Pediatría: Hospital San Juan de Dios (AMA

$M V)$.

Hospital Clínico San Borja-Arriarán (CLA, PS).

Hospital Dr. Exequiel González Cortés (CS, MZ)

Hospital Dr. Roberto Del Río (ST, JT)

Hospital Dr. Luis Calvo Mackenna (MES, MV)

Programa de Ginecología (SA)

Complejo Hospitalario Dr. Sótero Del Río, Santiago. (AB, TV).

Los autores no reportan conflictos de interés. Fuente de financiamiento: Proyecto FONDECYT 1040907

Recibido: 26 de agosto de 2011 Aceptado: 10 de febrero de 2012

Correspondencia a: M. Elena Santolaya de Pablo msantola@med.uchile.cl

\section{Introducción}

L a neutropenia febril (NF) es una complicación frecuente en los pacientes que reciben quimioterapia por cáncer. Las infecciones bacterianas, especialmente las bacteriemias, son la principal causa de morbi-mortalidad en niños con cáncer y $\mathrm{NF}^{1,2}$. En los años 70 y 80 se logró un gran impacto en disminuir la mortalidad asociada a NF, a través de la hospitalización y el uso precoz y empírico de antimicrobianos de amplio espectro $^{3-5}$. Esta estrategia se ha modificado en los últimos años con el conocimiento y validación de factores de riesgo que permiten clasificar las NF en aquellas que tienen alto y bajo riesgo de desarrollar una infección bacteriana invasora (IBI), implementando terapias antimicrobianas selectivas de acuerdo a los grupos de riesgo ${ }^{5-9}$.

Los microorganismos involucrados en la NF varían a lo largo del tiempo, habiendo predominado los bacilos gram- negativos (BGN) durante los años 70, para luego emerger las cocáceas grampositivas (CGP) en los 80, incluso sobrepasando a los BGN en la década de los $90^{2,10,11}$. Varios factores han influido en este cambio epidemiológico, entre ellos el uso de catéteres intravasculares, que favorecen las infecciones por Staphylococcus coagulasa negativa y otras CGP; la toxicidad sobre la mucosa oral de la quimioterapia que permite la translocación de Streptococcus del grupo viridans y el uso profiláctico de fluoroquinolonas que se relaciona con una disminución relativa de $\mathrm{BGN}^{11,12}$.

La etiología de los episodios de NF en niños con cáncer varía en diferentes regiones del mundo e incluso en los distintos hospitales de una misma región. Conocer los agentes etiológicos más frecuentes como también su susceptibilidad a los antimicrobianos en los episodios de $\mathrm{NF}$ es de gran importancia dado que permite escoger la terapia antimicrobiana empírica inicial de manera más racional. 
En Chile, Paya y cols., en representación del Comité de Infectología del Programa Infantil Nacional de Drogas Antineoplásicas (PINDA) publicaron el año 2001 la etiología y susceptibilidad antimicrobiana de los agentes causantes de infecciones del torrente circulatorio en niños con cáncer de cinco hospitales de Santiago (Región Metropolitana-RM) de Chile observada entre los años 1994 y 1998. Los resultados de este estudio confirmaron que en ese período predominaban Staphylococcus coagulasa negativa y Staphylococcus aureus, seguidos por Escherichia coli, Klebsiella spp y Pseudomonas spp., dando cuenta estas cinco especies de $82 \%$ de los aislados de sangre en esta población ${ }^{13}$.

El objetivo del presente estudio fue describir los agentes causantes de bacteriemia en los episodios de NF de alto riesgo (NFAR) de cursar con una IBI en niños con cáncer atendidos en los hospitales de la red PINDA de la RM, y comparar la frecuencia de los microorganismos aislados y su patrón de susceptibilidad en los dos períodos estudiados por nuestro grupo (1994-1998 vs 2004-2009).

\section{Pacientes y Métodos}

\section{Diseño global del estudio}

Estudio prospectivo, multicéntrico, de vigilancia epidemiológica de la etiología de los episodios de NFAR en niños con cáncer en tratamiento quimioterápico. Los pacientes analizados corresponden a niños bajo 18 años de edad, con cáncer y NFAR, admitidos entre mayo de 2004 y abril de 2009 en seis hospitales públicos de la RM de Chile pertenecientes a la red PINDA, como parte de Proyecto FONDECYT 1040907. Los hospitales que participaron brindan atención a aproximadamente 1.500.000 individuos bajo 18 años de edad, de las áreas sur, norte, occidente, central, sur-oriente y oriente de la RM y corresponden a los hospitales Dr. Exequiel González Cortés, Dr. Roberto Del Río, San Juan de Dios, San Borja-Arriarán, Dr. Sótero Del Río y Dr. Luis Calvo Mackenna, respectivamente. Todos los pacientes que consultaron por NF fueron invitados a participar en el proyecto FONDECYT señalado, se solicitó consentimiento informado a los padres o tutores legales y a los niños cuando tuvieron 11 años de edad o más. El proyecto fue aprobado por los comités de ética científicos de los respectivos Servicios de Salud.

A todos los pacientes con episodios de NFAR enrolados se les tomó muestras de sangre al ingreso para la realización de hemocultivos $(\mathrm{HC})$ automatizados, tanto de sangre periférica (2) como de cada lumen de catéter venoso central (CVC), cuando éste estaba implantado. Los episodios que cumplieron con la definición de $\mathrm{HC}$ positivos fueron incluidos en este análisis. Se describieron las características epidemiológicas generales de los pacientes con $\mathrm{HC}$ positivos y se compararon los hallazgos de los aislados en el período de estudio y su patrón de susceptibilidad, con los encontrados en un análisis previo realizado por el mismo grupo entre enero de 1994 y diciembre de 1998 en niños con cáncer, con o sin neutropenia, en cinco de los seis hospitales incluidos en el estudio actual.

\section{Estudio microbiológico}

En el período de análisis, todos los hospitales involucrados realizaron $\mathrm{HC}$ automatizados (BacT/Alert ${ }^{\circledR}$, bioMérieux, Inc, Durham, NC, USA). El volumen de sangre obtenido para cada muestra tuvo una relación 1:5 a 1:10, según la edad del paciente, de acuerdo con la recomendación del método BacT/Alert ${ }^{\circledR}$.

El estudio de susceptibilidad fue realizado por método de difusión en agar (Kirby Bauer) con puntos de corte establecidos por el CLSI vigente. Los antimicrobianos incluidos en el estudio de CGP fueron oxacilina, vancomicina, amikacina y cefotaxima; en el estudio de BGN: gentamicina, amikacina, cefotaxima, ceftriaxona, ciprofloxacina, imipenem y meropenem y en BGN no fermentadores: gentamicina, amikacina, ceftazidima, cefepime, ciprofloxacina, imipenem y meropenem.

\section{Definiciones}

Neutropenia febril de alto riesgo: neutropenia (RAN $\leq$ a 500 céls $/ \mathrm{mm}^{3}$ ) más fiebre (una medición de temperatura axilar $>38,5^{\circ} \mathrm{C}$ o dos mediciones $>38^{\circ} \mathrm{C}$ espaciadas por al menos una hora); y uno de los siguientes factores de riesgo de IBI: leucemia en recaída, hipotensión arterial, proteína $\mathrm{C}$ reactiva $(\mathrm{PCR}) \geq 90 \mathrm{mg} / \mathrm{L}$, o la presencia de los dos siguientes factores de riesgo: recuento de plaquetas $<50.000 / \mathrm{mm}^{3}$ y número de días entre el término del último ciclo de quimioterapia y el inicio del actual episodio de fiebre $\leq$ a $7^{14}$.

Hemocultivo positivo: Se consideró que un $\mathrm{HC}$ era positivo si hubo un aislamiento único de cualquier patógeno reconocido, excepto para Staphylococcus coagulasa negativa, en cuyo caso se exigió que hubiese dos $\mathrm{HC}$ positivos para este agente, en un período menor a 20 horas de incubación. Si se aisló Staphylococcus coagulasa negativa en un HC y otra especie diferente en el segundo, o Staphylococcus coagulasa negativa con susceptibilidad diferente en los dos $\mathrm{HC}$ obtenidos, se interpretó como una contaminación. Se consideró un agente por cada episodio de $\mathrm{NF}^{15,16}$.

\section{Análisis estadístico}

Se elaboró una base de datos con el programa Microsoft Excel 2010 y se utilizó el programa Stata 10.0 para realizar el análisis estadístico. Los resultados de las variables cuantitativas se expresaron en números absolutos y porcentajes. En el análisis comparativo de la etiología y susceptibilidad antimicrobiana de los episodios de NFAR del período 2004-2009 versus los del período 


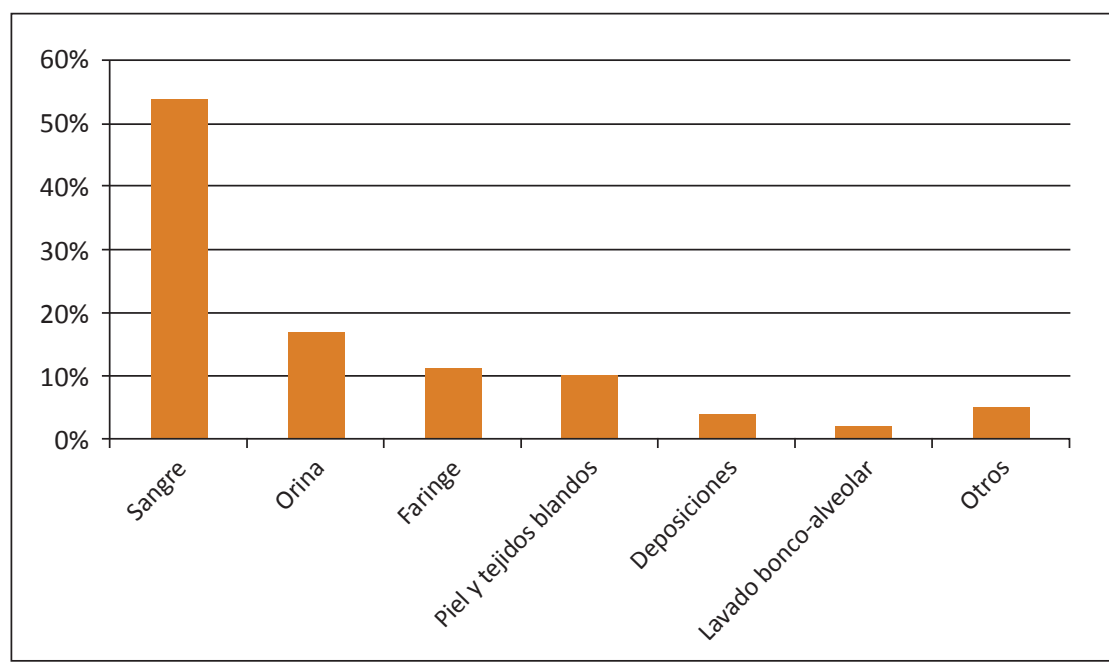

Figura 1. Sitios de aislamiento microbiológico en 839 episodios de neutropenia febril de alto riesgo en niños con cáncer en seis hospitales de la Región Metropolitana, período 2004-2009.

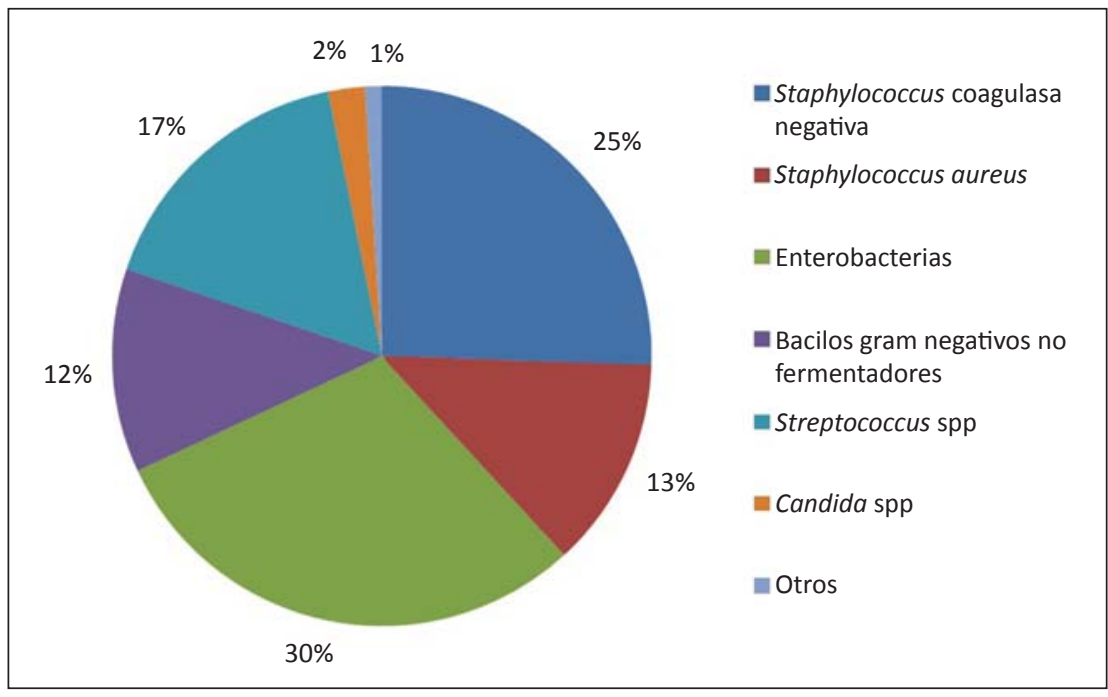

Figura 2. Frecuencia relativa de microorganismos aislados en 181 hemocultivos de niños con cáncer y neutropenia febril de alto riesgo, en seis hospitales de la Región Metropolitana, período 2004-2009.

1994-1998, los datos se expresaron en promedios más intervalos de confianza de $95 \%$, utilizando los métodos de Wald y Agresti, según necesidad.

\section{Resultados}

Se documentaron 839 episodios de NFAR entre mayo de 2004 y abril de 2009. Hubo detección de microorganismos en 335 episodios (40\%), siendo la sangre el sitio de mayor frecuencia de aislamiento, en 181/335 episodios (54\%) (Figura 1).
Los 181 microorganismos aislados desde HC correspondieron a $101 \mathrm{CGP}(56 \%), 76 \mathrm{BGN}(42 \%)$ y 4 levaduras (2\%). Para facilitar el análisis de susceptibilidad, las cepas encontradas se agruparon en CGP: Staphylococcus coagulasa negativa (n: 46), Streptococcus spp (n: 30) (S. grupo viridans 26, S. pneumoniae 3 , S. no hemolítico 1), S. aureus (n: 23), Enterococcus spp. (n: 1), Staphylococcus haemolyticus 1 (n: 1); BGN: Enterobacterias (n: 54) (Escherichia coli 36, Enterobacter spp 9, Klebsiella spp 8 y Citrobacter spp. 1); BGN no fermentadores (n: 22) (Pseudomonas spp. 17, Acinetobacter spp. 4, otro 1) y Candida spp. 4 (Figura 2). Cinco especies representaron más de $80 \%$ de los microorganismos aislados en esta población: Staphylococcus coagulasa negativa $(25 \%)$, E. coli $(20 \%)$, Streptococcus grupo viridans (14\%), S. aureus (13\%) y Pseudomonas spp (9\%). No hubo diferencias significativas en la frecuencia de microorganismos aislados por hospital.

Al comparar la frecuencia relativa de los microorganismos aislados en el período 2004-2009 con el período 1994-1998, hubo una disminución significativa de Staphylococcus coagulasa negativa, desde $44 \%$ en el período 1994-1998 (IC 95\%: 40,2-47,5) a 25\% en el período 2004-2009 (IC 95\%:19,1-31,8) y un aumento significativo de Streptococcus spp, desde 4\% en el período 1994-1998 (IC95\% 2,9-5,9) a 17\% en el período 2004-2009 (IC95\% 11,2-22). El resto de los microorganismos no presentaron cambios significativos en su frecuencia (Tabla 1).

En 151/177 cepas bacterianas $(85,3 \%)$ se obtuvo estudio de susceptibilidad a diferentes antimicrobianos, comparable con el realizado en el período previo (19941998). En la Tabla 2 se muestra como Staphylococcus coagulasa negativa aumentó significativamente la resistencia a oxacilina cuando se comparan los períodos 1994-1998 y 2004-2009: 55\% (IC95\%: 46,7-62,6) y $77 \%$ (IC95\%: 64,1-89,4), respectivamente. Por otra parte, la resistencia de $S$. aureus a oxacilina fue de $31 \%$ en el período 1994-1998, lo que disminuyó a 14\% en el período 2004-2009, diferencia que no alcanzó significancia estadística. Ni Staphylococcus coagulasa negativa ni S. aureus presentaron resistencia a vancomicina en ambos períodos estudiados. La resistencia a penicilina de Streptococcus spp aumentó de manera no significativa desde el período 1994-1998 al período 2004-2009 (50 vs 75\%), sin resistencia a vancomicina.

El análisis de susceptibilidad global de las enterobacterias encontradas, como también la comparación con el estudio previo se muestran en la Figura 3. Destaca una disminución no significativa de la sensibilidad a cefotaxima y ceftriaxona, mantención de sensibilidad en el tiempo sobre $80 \%$ a aminoglucósidos, y cercana a $100 \%$ a imipenem.

La Figura 4 muestra el estudio de susceptibilidad de los BGN no fermentadores identificados, que representa 14 
Tabla 1. Frecuencia relativa de los microorganismos aislados desde hemocultivos en niños con cáncer y episodios de neutropenia febril, períodos 1994-1998 y 2004-2009

\begin{tabular}{|c|c|c|c|c|c|c|}
\hline \multirow[t]{2}{*}{ Microorganismo } & \multicolumn{3}{|c|}{ 1994-1998 } & \multicolumn{3}{|c|}{ 2004-2009 } \\
\hline & $\mathbf{n}$ & $\%$ & IC95\%* & $\mathbf{n}$ & $\%$ & IC95\%* \\
\hline Staphylococcus coagulasa negativa & 310 & $44^{* *}$ & $40,2-47,5$ & 46 & $25^{* *}$ & $19,1-31,8$ \\
\hline Staphylococcus aureus & 118 & 17 & $13,9-19,4$ & 23 & 13 & $7,9-17,6$ \\
\hline Streptococcus spp. & 31 & $4^{* *}$ & $2,9-5,9$ & 30 & $17^{* *}$ & $11,2-22,0$ \\
\hline Enterobacterias & 146 & 21 & $17,7-23,6$ & 54 & 30 & $22,7-35$, \\
\hline Bacilos gramnegativos no fermentadores & 44 & 6 & $4,4-8,0$ & 22 & 12 & $6,9-16,3$ \\
\hline Hongos & 28 & 4 & $2,5-5,4$ & 4 & 2 & $0,1-4,4$ \\
\hline Otros & 30 & 4 & $2,7-5,3$ & 2 & 1 & $0,1-2,3$ \\
\hline Total & 707 & & & 181 & & \\
\hline
\end{tabular}

Tabla 2. Resistencia a antimicrobianos de bacterias aisladas desde hemocultivos en niños con cáncer, períodos 1994-1998 y 2004-2009

\begin{tabular}{|c|c|c|c|c|c|}
\hline \multirow[t]{2}{*}{ Microrganismo } & \multirow[t]{2}{*}{ Antimicrobiano } & \multicolumn{2}{|c|}{ 1994-1998 } & \multicolumn{2}{|c|}{ 2004-2009 } \\
\hline & & $\%$ & IC $95 \%$ & $\%$ & IC $95 \%$ \\
\hline Staphylococcus coagulasa negativa & oxacilina**** & 55 & $46,7-62,6$ & 77 & $64,1-89,4$ \\
\hline Staphylococcus aureus & oxacilina & 31 & $23,0-39,7$ & 14 & $0-28,0$ \\
\hline Streptococcus grupo viridans & penicilina & 50 & $18,8-81,2^{*}$ & 75 & $40,0-93,7^{*}$ \\
\hline \multirow[t]{5}{*}{ Enterobacterias** } & gentamicina & 19 & $12,8-25,0$ & 19 & $7,0-30,2$ \\
\hline & amikacina & 18 & $11,9-23,8$ & 16 & $5,0-26,2$ \\
\hline & cefotaxima & 25 & $18,4-32,3$ & 35 & $21,0-48,6$ \\
\hline & ceftriaxona & 23 & $11,9-33,7$ & 33 & $16,5-50,0$ \\
\hline & imipenem & 2,4 & $0-7,2$ & 2,7 & $0-7,9$ \\
\hline \multirow[t]{5}{*}{ Bacilos gramnegativos no fermentadores*** } & gentamicina & 24 & $9,3-37,8$ & 27 & $4,3-49,1$ \\
\hline & amikacina & 9,3 & $0,6-18,0$ & 25 & $3,8-46,2$ \\
\hline & ceftazidima & 19 & $5,5-33,3$ & 31 & $8,5-54,0$ \\
\hline & ciprofloxacina & 11 & $0-24,3$ & 19 & $0-37,9$ \\
\hline & imipenem & 6,7 & $0-19,3$ & 14 & $0-32,6$ \\
\hline $\begin{array}{l}\text { *Todos los intervalos son calculados por mét } \\
\text { **Enterobacterias: Escherichia coli, Enterobac } \\
\text { *** Bacilos gramnegativos no fermentadores: } \\
\text { ****Diferencia significativa en la resistencia d }\end{array}$ & $\begin{array}{l}\text { o de Wald excepto } \\
\text { r spp, Klebsiella sp } \\
\text { eudomonas spp., } \\
\text { Staphylococcus co }\end{array}$ & $\begin{array}{l}\text { lado } \\
\text { spp. } \\
\text { spp. }\end{array}$ & gresti. & & \\
\hline
\end{tabular}

cepas de Pseudomonas spp y 2 de Acinetobacter baumannii, con sensibilidad estable a gentamicina y disminución de sensibilidad, en forma no significativa, a amikacina, ceftazidima, ciprofloxacina e imipenem.

\section{Discusión}

Conocer la etiología de los episodios de NFAR en pacientes con cáncer tiene importancia para implementar tratamientos antimicrobianos ajustados a la epidemiolo- gía de cada establecimiento, lo que tiene impacto en la morbilidad y mortalidad.

Las infecciones del torrente sanguíneo son la forma más común de infección en los episodios de NF, con una positividad de los $\mathrm{HC}$ que va de 20 a 35\% según distintas series $^{17,18}$. Las bacterias son los agentes etiológicos más frecuentemente involucrados en el inicio de los episodios de NF. Ambos hechos se confirman en nuestro estudio, con $22 \%$ (181/839) de HC positivos, de los cuales $98 \%$ correspondieron a bacterias. 


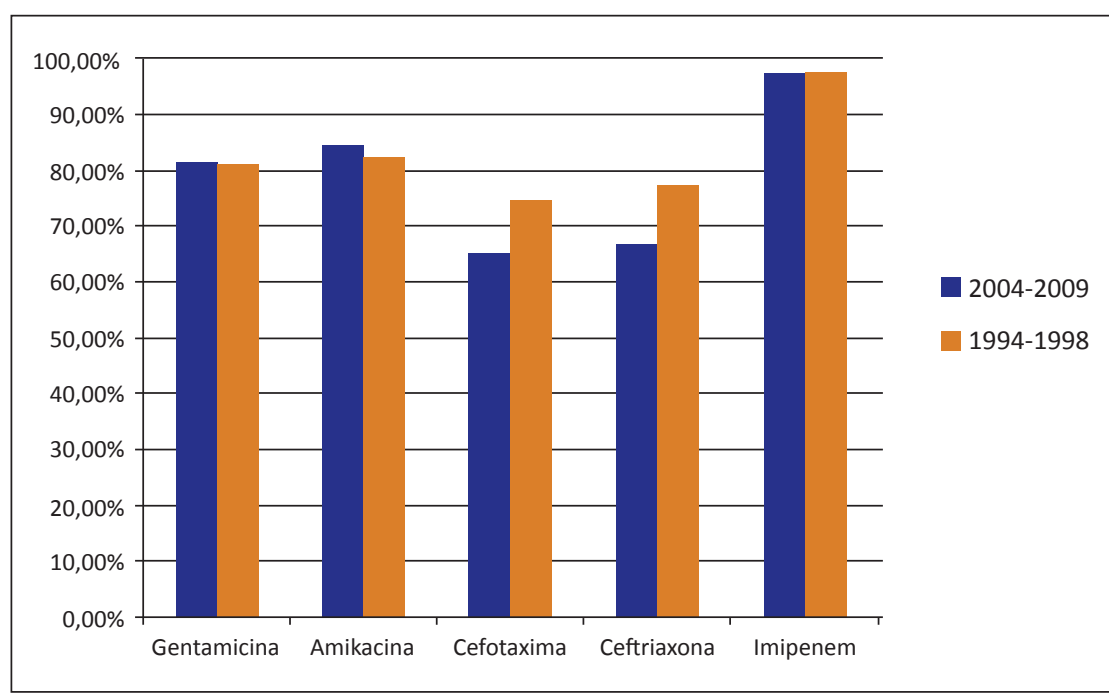

Figura 3. Susceptibilidad in vitro a antimicrobianos de enterobacterias aisladas desde hemocultivos en niños con cáncer, período 1994-1998 y 2004-2009.

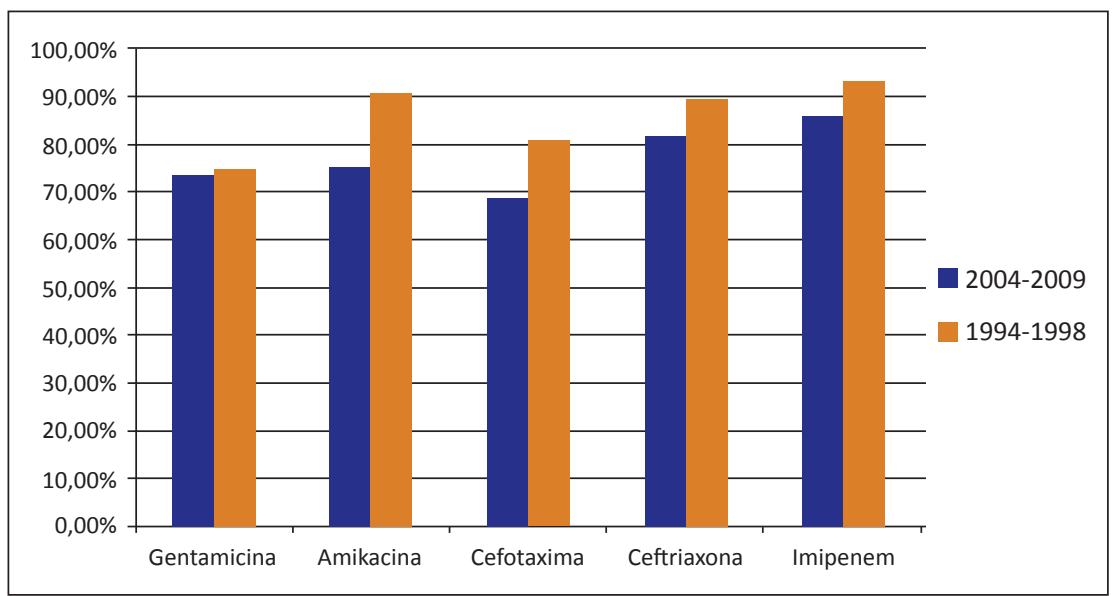

Figura 4. Susceptibilidad in vitro a antimicrobianos de bacilos gramnegativos no fermentadores aislados desde hemocultivos en niños con cáncer, período 1994-1998 y 2004-2009.

Algunas publicaciones recientes analizan el espectro de los patógenos aislados en las bacteriemias de pacientes con cáncer y NF, mostrando una tendencia al aumento de las CGP, tanto en series pediátricas como de adultos ${ }^{2,18-24}$, siendo los microorganismos aislados con mayor frecuencia Staphylococcus coagulasa negativa y Streptococcus grupo viridans ${ }^{23,25-29}$ en porcentajes que van de 57 a $80 \% \%^{1,21}$. En nuestro estudio, la frecuencia de CGP fue 56\%, similar a lo descrito internacionalmente; posiblemente esta frecuencia sea atribuible al alto número de niños portadores de CVC y al uso de quimioterapias más agresivas con mayor incidencia de mucositis. Es necesario precisar el no uso de profilaxis con quinolonas (ciprofloxacina) en los hospitales participantes en este estudio. El porcentaje predominante de aislados de CGP contrasta con otros estudios en que hay mayor frecuencia de BGN, tanto en series nacionales como extranjeras ${ }^{14,16,24,30-34}$.

Estas diferencias ponen de manifiesto la necesidad del conocimiento institucional de los agentes causantes de bacteriemia prevalentes en los episodios de NFAR y su susceptibilidad, objetivo principal de nuestro estudio. En el período 2004-2009, la distribución global de las cepas por especie confirma el predominio de Staphylococcus coagulasa negativa, seguido por E. coli y Streptococcus grupo viridans, grupo de bacterias que supera a Klebsiella spp, S. aureus y Pseudomonas spp, en relación al período anterior, en que ocupaba el octavo lugar en frecuencia. Una probable razón para el aumento relativo de Streptococcus grupo viridans es el mayor daño de mucosas en pacientes tratados con citarabina, la que es utilizada en protocolos de leucemia mieloide aguda, razón por la que en algunos centros extranjeros se recomienda el uso empírico de vancomicina en episodios de NF en pacientes bajo este régimen terapéutico ${ }^{29}$.

La comparación de susceptibilidad antimicrobiana entre los dos períodos analizados tiene algunas limitaciones. En el período 1994-1998 se evaluó la etiología de las bacteriemias en niños con cáncer, con y sin neutropenia, y en el periodo actual sólo en pacientes con neutropenia febril de alto riesgo. Reconociendo esta limitación en las poblaciones analizadas, no obstante, la comparación nos parece útil para el manejo clínico, considerando que el aislamiento de bacterias en sangre es mínimo en los episodios de NF de bajo riesgo como en los episodios febriles sin neutropenia, por lo que el alto porcentaje de bacterias aisladas de hemocultivos en niños con cáncer provienen de pacientes con episodios de NFAR.

La única diferencia estadísticamente significativa encontrada entre ambos períodos fue el aumento de la resistencia de Staphylococcus coagulasa negativa a oxacilina, asociada probablemente al uso rutinario de cloxacilina en los esquemas iniciales de manejo de NFAR, especialmente en paciente portadores de CVC. Es destacable la permanencia en el tiempo de la sensibilidad de enterobacterias a aminoglucósidos y carbapenémicos, con un alza de resistencia a cefalosporinas de $3^{\mathrm{a}}$ generación, pero sin significancia estadística. Como otra limitación de nuestro estudio, no contamos con información sostenida en todo el período de estudio sobre la producción de $\beta$-lactamasas de espectro extendido, práctica habitual en los laboratorios de Microbiología en la actualidad.

La mantención de la susceptibilidad a los antimicrobianos en el tiempo, da cuenta de su uso racional en los hospitales del grupo PINDA que manejan a los niños con cáncer y NF, de acuerdo a criterios validados de alto o bajo riesgo de IBI, suscribiendo el consenso nacional de manejo de estos pacientes ${ }^{9,17}$ y el consenso latinoamericano de manejo de la NF en niños con cáncer ${ }^{35}$. Sin embargo, al 
analizar la susceptibilidad de BGN no fermentadores, si bien no hubo diferencias significativas en los dos períodos comparados, se observó una tendencia a la disminución de sensibilidad en los diferentes grupos de antimicrobianos observados, lo que nos parece un hallazgo preocupante que obliga a una vigilancia estrecha. Una limitante en la interpretación de los datos de susceptibilidad es el número reducido de cepas de algunos de los microorganismos analizados, lo que podría influir en que no se alcanzó significancia estadística en las comparaciones o en los intervalos de confianza muy amplios mostrados en la Tabla 2.

El análisis final de nuestros datos pone de manifiesto la necesidad de realizar una vigilancia epidemiológica activa de los microorganismos involucrados en las bacteriemias de los niños con cáncer y episodios de NF, para poder ofrecer el mejor tratamiento antimicrobiano empírico inicial a estos pacientes, en términos de eficacia, proporcionalidad a la estratificación de riesgo y a la epidemiología local ${ }^{36,37}$. Desde la perspectiva de los hospitales involucrados en este estudio, los datos encontrados avalan la necesidad de mantener una terapia empírica inicial que cubra tanto BGN como CGP.

\section{Resumen}

Introducción: Conocer la etiología de los episodios de neutropenia febril de alto riesgo (NFAR) en pacientes con cáncer tiene importancia para implementar tratamientos antimicrobianos ajustados a la epidemiología local, lo que tiene impacto en la morbilidad y mortalidad. Objetivo: Describir la etiología de las bacteriemias en niños con cáncer y NFAR en el período 2004-2009, en la red PINDA de Santiago (Región Metropolitana), Chile, y comparar estos agentes y su susceptibilidad antimicrobiana con un estudio previo realizado en el período 1994-1998. Material y Métodos: Se registraron prospectivamente los agentes causantes de bacteriemia y su susceptibilidad a antimicrobianos de los pacientes bajo 18 años de edad en tratamiento quimioterápico por cáncer, ingresados con diagnóstico de NFAR a los seis hospitales de la red, durante el período 2004-2009. Resultados: De 839 episodios de NFAR, 181 tuvieron hemocultivos positivos, correspondientes a cocáceas grampositivas $(56 \%)$, bacilos gramnegativos (42\%) y levaduras $(2 \%)$. Los agentes más frecuentemente aislados fueron: Staphylococcus coagulasa negativa (25\%), Escherichia coli (20\%), Streptococcus grupo viridans (14\%), Staphylococcus aureus (13\%) y Pseudomonas spp (9\%). Al comparar los dos períodos de tiempo, destacan los siguientes cambios significativos: disminución en frecuencia relativa de Staphylococcus coagulasa negativa (desde 44 a 25\%), aumento de Streptococcus spp (desde 4 a 17\%), y aumento de la resistencia de Staphylococcus coagulasa negativa a oxacilina (desde 55 a 77\%). Conclusiones: Se dan a conocer los principales agentes etiológicos de los episodios de NFAR y la susceptibilidad a antimicrobianos en un período de cinco años. Esto permite racionalizar el manejo antimicrobiano empírico de los episodios de NFAR en esta población.

\section{Referencias}

1.- Castagnola E, Fontana V, Caviglia I, Caruso S, Faraci M, Fioredda F, et al. A prospective study on the epidemiology of febrile episodes during chemotherapy-induced neutropenia in children with cancer or after hemopoietic stem cell transplantation. Clin Infect Dis 2007; 45: 1296-1304.

2.- Hakim H, Flynn P, Knapp K, Srivastava D, Gaur A. Etiology and clinical course of febrile neutropenia in children with cancer. J Pediatr Hematol Oncol 2009; 31: 623-9.

3.- Pizzo P A, Robichaud K J, Wesley R, Commers J R. Fever in the pediatric and young adult patient with cancer: A prospective study of 1001 episodes. Medicine (Baltimore) 1982; 61: 153-65.

4.- Pizzo P A, Rubin M, Freifeld A,Walsh T J. The child with cancer and infection. I. Empiric therapy for fever and neutropenia, and preventive strategies. J Pediatr 1991; 119 : 679-94.

5.- Hughes W T, Armstrong D, Bodey G P, Bow E J, Brown A E, Calandra T, et al.
Guidelines for the use of antimicrobial agents in neutropenic patients with cancer. Clin Infect Dis 2002; 34: 7 30-51.

6.- Paganini H, Gómez S, Ruvinsky S, Zubizarreta P, Latella A, Fraquelli L, et al. Outpatient, sequential, parenteral-oral antibiotic therapy for lower risk febrile neutropenia in children with malignant disease: a single-center, randomized, controlled trial in Argentina. Cancer 2003; 97 : 1775-80.

7.- Kamana M, Escalante C, Mullen C A, FrisbeeHume S, Rolston K V. Bacterial infections in low-risk, febrile neutropenic patients. Cancer 2005; 104: 422-6.

8.- Santolaya M E, Álvarez A M, Aviles C L, Becker A, Cofré J, Cumsille M, et al. Early hospital discharge followed by outpatient management versus continued hospitalization of children with cancer, fever and neutropenia at low risk for invasive bacterial infection. J Clin Oncol 2004; 22: 3784-9.

9.- Santolaya M E, Álvarez A M, Avilés C L, Becker A, Cofré J, Enríquez N, et al. Prospective evaluation of a model of prediction of invasive bacterial infection risk among children with cancer, fever and neutropenia. Clin Infect Dis 2002; 35: 678-83.

10.- Aquino V, Pappo A, Buchanan G, Tkaczewski I, Mahmoud M. The changing epidemiology of bacteremia in neutropenic children with cancer. Pediatr Infect Dis J 1995; 14: 140.

11.- Ramphal R. Changes in the etiology of bacteremia in febrile neutropenic patients and the susceptibilities of the currently isolated pathogens. Clin Infect Dis 2004; 39: S25-31.

12.- Cordonnier C, Buzyn A, Leverger G, Herbrecht R, Hunault M, Leclercq R, et al Epidemiology and risk factors for grampositive coccal infections in neutropenia: toward a more targeted antibiotic strategy. Clin Infect Dis 2003; 36: 149-58.

13.- Payá E, Álvarez A, Avilés C, Cofré J, Enríquez N, Salgado C, et al. Agentes causantes de infecciones del torrente circulatorio en niños con cáncer, en cinco hospitales de Santiago (1994-1998). Rev Med Chile 2001; 129: 1297-304.

14.- Santolaya M E, Álvarez A M, Becker A, Cofré J, Enríquez N, O’Ryan M, et al. 
Prospective, multicenter evaluation of risk factors associated with invasive bacterial infection in children with cancer, neutropenia, and fever. J Clin Oncol 2001; 19: 3415-21.

15.- Kassis C, Rangaraj G, Jiang Y, Hachem R Y, Raad I. Differentiating culture samples representing coagulase-negative staphylococcal bacteremia from those representing contamination by use of time-to-positivity and quantitative blood culture methods. J Clin Microbiol 2009; 47: 3255-60.

16.- Rabagliati R, Fuentes G, Orellana E, Oporto J, Domínguez I, Benítez R, et al. Etiología de episodios de neutropenia febril en pacientes adultos con cáncer hematológico y de órganos sólidos en el Hospital Clínico Universidad Católica, Santiago-Chile. Rev Chil Infectol 2009; 26: 106-13

17.- Santolaya M E, Rabagliati R, Bidart T, Paya E, Guzmán A M, Morales R, et al. Consenso: Manejo racional del paciente con cáncer, neutropenia y fiebre. Rev Chil Infectol 2005; 22: S79-S113.

18.- Yadegarynia D, Tarrand J, Raad I, Rolston K. Current spectrum of bacterial infections in patients with cancer. Clin Infec Dis 2003; 37: 1144-5.

19.- Klastersky J, Ameye L, Maertens J, Georgala A,Muanza $\mathrm{F}$ et al. Bacteraemia in febrile neutropenic cancer patients. Int J Antimicrob Agents 2007; 30: S51-S59.

20.- Viscoli C, Varnier O, Machetti M. Infections in patients with febrile neutropenia: epidemiology, microbiology, and risk stratification. Clin Infect Dis 2005; 40: S240-S245.

21.- Wisplinghoff $H$, Seifert $H$, Wenzel RP, Edmond MB. Current trends in the epidemiology of nosocomial bloodstream infections in patients with hematological malignancies and solid neoplasms in hospitals in the United States. Clin Infect Dis 2003; 36: 1103-10.
22.- Lehrnbecher T, Varwig D, Kaiser J, Reinhardt D, Klingebiel T, Creutzig U. Infectious complications in pediatric acute myeloid leukemia: analysis of the prospective multiinstitutional clinical trial AMLBFM 93. Leukemia Jan 2004; 18: 72-7.

23.- Bochud P Y, Calandra T, Francioli P. Bacteremia due to viridans streptococci in neutropenic patients: a review. Am J Med 1994; 97: 256-64.

24.- Greenberg D, Moser A, Yagupsky P, Peled N, Hofman Y, Kapelushnik J, et al. Microbiological spectrum and susceptibility patterns of pathogens causing bacteraemia in paediatric febrile neutropenic oncology patients: comparison between two consecutive time periods with use of different antibiotic treatment protocols. Int J Antimicrob Agents. 2005; 25 : 469-73.

25.- Paganini H, Staffolani V, Zubizarreta P, Casimir L, Lopardo H, Luppino V. Viridans streptococci bacteraemia in children with fever and neutropenia: a case-control study of predisposing factors. Eur J Cancer 2003; 39: 1284-9.

26.- Reilly A F, Lange B J. Infections with viridans group streptococci in children with cancer. Pediatr Blood Cancer 2007; 49 (6): 774-80.

27.- Okamoto Y, Ribeiro R C, Srivastava D K, Shenep J L, Pui C H, Razzouk B I. Viridans streptococcal sepsis: clinical features and complications in childhood acute myeloid leukemia. J Pediatr Hematol Oncol 2003; 25: 696-703.

28.- Shenep J L. Viridans-group streptococcal infections in immunocompromised hosts. Int $\mathrm{J}$ Antimicrob Agents 2000; 14: 129-35.

29.- Rossetti F, Cesaro S, Putti M C, Zanesco L. High-dose cytosine arabinoside and viridans streptococcus sepsis in children with leukemia. Pediatr Hematol Oncol 1995; 12: 387-92.

30.- Ariffin H, Navaratnam P, Lin HP. Surveillance study of bacteraemic episodes in febrile neutropenic children. Int J Clin Pract May 2002; 56: $237-40$

31.- Velasco E, Byington R, Martins C S, Schirmer M, Dias LC, Goncalves VM. Bloodstream infection surveillance in a cancer centre: a prospective look at clinical microbiology aspects. Clin Microbiol Infect 2004; 10 : 542-9.

32.- Paul M, Gafter-Gvili A, Leibovici L, Bishara J, Levy I, Yaniv I, et al. The epidemiology of bacteremia with febrile neutropenia: experience from a single center, 1988-2004. Isr Med Assoc J 2007; 9: 424-9.

33.- Gaytan-Martínez J, Mateos-García E, SánchezCortes E, González-Llaven J, Casanova-Cardiel L J, Fuentes-Allen J L. Microbiological findings in febrile neutropenia. Arch Med Res 2000; 31: 388-92.

34.- Kanafani Z A, Dakdouki G K, El-Chammas K I, Eid S, Araj G F, Kanj S S. Bloodstream infections in febrile neutropenic patients at a tertiary care center in Lebanon: a view of the past decade. Int J Infect Dis 2007; 11: 450-3.

35.- Paganini H, Santolaya M E, Álvarez M, Araña $\mathrm{R}$, de Jesús M, Arteaga R, et al. Diagnóstico $\mathrm{y}$ tratamiento de la neutropenia febril en niños con cáncer. Consenso de la Sociedad Latinoamericana de Infectología Pediátrica. Rev Chil Infectol 2011; 28 (Supl 1): S10-S38.

36.- Freifeld A, Bow E, Sepkowitz K, Boeckh M J, Ito J, Mullen C A, et al. Clinical practice guideline for the use of antimicrobial agents in neutropenic patients with cancer: 2010 Update by the Infectious Diseases Society of America. Clin Infect Dis 2011; 52(4): e56-e93.

37.- Palazzi D L. The use of antimicrobial agents in children with fever during chemotherapyinduced neutropenia: the importance of risk stratification. Ped Infect Dis J 2011; 30: 887-90. 